\begin{tabular}{|c|l|}
\hline Title & Zn protoporphyrin IX is formed not from heme but from protoporphyrin IX \\
\hline Author(s) & Wakamatsu, Jun-ichi; Okui, Jun; Hay ashi, Nobutaka; Nishimura, Takanori; Hattori, A kihito \\
\hline Citation & $\begin{array}{l}\text { Meat Science, 77(4), 580-586 } \\
\text { https://doi.org/10.1016/.meatsci.2007.05.008 }\end{array}$ \\
\hline Issue Date & $2007-12$ \\
\hline Doc URL & http://hdl.handle.net/2115/32300 \\
\hline Type & article (author version) \\
\hline File Information & MEA TSCI-00261.pdf \\
\hline
\end{tabular}

Instructions for use 
3

17 Faculty of Agriculture, Hokkaido University

18 N-9 W-9, Kita-ku, Sapporo, Hokkaido 060-8589, Japan but from protoporphyrin IX.

* Corresponding author.

Tel.: +81 11706 2547; Fax.: +81 117062547

E-mail address: jwaka@anim.agr.hokudai.ac.jp (J. Wakamatsu)

\section{Zn protoporphyrin IX is formed not from heme}

Jun-ichi WAKAMATSU*, Jun OKUI, Nobutaka HAYASHI, Takanori NISHIMURA, Akihito HATTORI

Division of Bioresource and Product Science, Graduate School of Agriculture, Hokkaido University, Sapporo, Hokkaido 060-8589, Japan

Meat Science Laboratory, Division of Bioresource and Product Science, Research 
21 We examined the effects of exogenous myoglobin, a bivalent chelator, and nitrite on Zn protoporphyrin IX (ZPP) formation by using model systems. ZPP was formed in a 23 model solution without addition of exogenous myoglobin. After incubation, the amount 24 of ZPP in a model solution was increased but that of heme was not decreased compared with the amounts before incubation. Protoporphyrin IX (PPIX) instead of ZPP also accumulated in a model solution with addition of EDTA, but the amount of heme was

27 not reduced. These results suggested that ZPP was not formed by the Fe-Zn 28 substitution in heme but was formed by the insertion of $\mathrm{Zn}$ into PPIX, which was 29 formed independently. The fact that the effects of various factors in model systems with/without addition of a bivalent chelator were similar suggested that ZPP formation was strongly affected by PPIX formation. Inhibition of PPIX formation by nitrite 32 might be the reason for the low levels of ZPP in cured meats.

33

34 Keywords: Zn protoporphyrin IX; protoporphyrin IX; color; dry-cured meat products; nitrite; nitric oxide 
Dry-cured meat products have been produced and consumed throughout history by a diversity of cultures in different parts of the world. Parma ham, one of the Italian dry-cured hams, is made from heavier pigs (> $150 \mathrm{~kg}$ in liveweight) and is processed for at least 12 months without addition of nitrite/nitrate (Toldrá, 2002). It has been reported that Parma ham has a red porphyrin derivative that is not nitrosylmyoglobin or oxymyoglobin and is extractable by water, acetone/water $(75 \% / 25 \%)$ and tetrahydrofuran (Morita, Niu, Sakata \& Nagata, 1996; Møller, Adamsen \& Skibsted, 2003; Parolari, Gabba \& Saccani, 2003). The pigment was purified and identified to be Zn protoporphyrin IX (ZPP) by ESI-HR-MS analysis (Wakamatsu, Nishimura \& Hattori, 2004). Mor eover, the presence of zinc in the red pigment was directly demonstrated by scanning electron microscopy/energy dispersive X-ray microanalysis (SEM-EDX) (Wakamatsu, Ito, Nishimura \& Hattori, 2007). Møller, Adamsen, Catharino, Skibsted, \& Eberlin (2007) showed by using ESI-MS and TOF-MS analysis that zinc protoporphyrin IX is present not only in Parma ham but also in Iberian ham.

Effects of various factors on ZPP formation have been investigated using a model system, and it has been shown that oxygen inhibited ZPP formation and that ZPP was formed in the absence of microorganisms (Wakamatsu, Okui, Nishimura \& Hattori, 2004). It has also been reported that the amount of ZPP and protoporphyr in IX (PPIX) were increased in pork and turkey during anaerobic conditions (Veberg et al., 2006). By use of autofluor escence of ZPP, it has been revealed that ZPP was distributed in not only lean meat but also fat tissue by using near-UV purple LED irradiation and an image analysis (Wakamatsu, Odagiri, Nishimura \& Hattori, 2006).

On the other hand, exogenous myoglobin was added into a model solution that was established by Wakamatsu, Okui, Nishimura \& Hattori (2004). The addition of exogenous myoglobin may affect ZPP formation. Moreover, the amount of ZPP formed 
has only been measured by fluorescence intensity, and the amount of heme has not been

64 determined at all. In order to elucidate the mechanism by which ZPP is formed, these

65 porphyrins must be quantitatively determined. A recent study has shown that ZPP is

66 present not only in Parma ham but also in Iberian ham but that ZPP content in meat

67 products cured with nitrite is very low (Adamsen Møller, Laursen, Olsen \& Skibsted,

68 2006). It is still not clear why ZPP content in cured meat products is much lower than

69 that in dry-cured ham without addition of nitrite.

70 In this study, to elucidate the mechanism by which ZPP is formed, we investigated

71 the effects of various factors on ZPP formation by using a simpler model system with no

72 addition of exogenous myog lobin and a new model system in which PPX was formed by

73 the addition of ethylenediaminetetraacetate (EDTA). Then we measured the contents

74 of heme, ZPP and PPIX before and after incubation in these model solutions and

75 investigated stoichiometrically the mechanism by which ZPP or PPIX was formed.

76 Additionally, we investigated the inhibitory effect on ZPP formation of nitrite, which is

77 generally used in cured meat products. 


\subsection{Materials}

81 (from horse skeletal muscle), penicillin G potassium, streptomycin sulfate and gentamicin sulfate were purchased from Wako Pure Chemical Industries, Ltd. (Osaka, Japan). Acetone, acetic acid, acetate ethyl, methanol and ammonium acetate were purchased from Kanto Chemical Co. Inc. (Tokyo, Japan). Methanol was of HPLC grade and other chemicals were of analytical grade.

\subsection{Model system}

90 homogenized with 2 volumes of distilled water using a ho mogenizer at 10,000 rpm for 1 min. Antibiotics were added to the model solutions to final concentrations of 100 units $/ \mathrm{ml}$ for penicillin $\mathrm{G}$ potassium, $0.1 \mathrm{mg} / \mathrm{ml}$ for streptomycin sulfate and $0.05 \mathrm{mg} / \mathrm{ml}$ for gentamicin sulfate. The solutions were put into gas-impermeable bags and incubated at $25{ }^{\circ} \mathrm{C}$ for 5 days in darkness using an environmental chamber. An anaerobic condition was obtained by using a commercial kit (Oxygen Absorbing System; I.S.O. Inc., Yokohama, Japan) consisting of an oxygen absorber (A-500HS, I.S.O. Inc.) and an oxygen indicator tablet. The color of the oxygen indicator tablet changes from violet to pink when the oxygen concentration becomes less than $0.1 \%$.

102

103

\subsection{Fluorescent analysis}

104 

Wakamatsu, Okui, Nishimura \& Hattori (2004) with minor modification. After extraction by acetone, the fluorescent spectra of the extracts were measured from 450 to $700 \mathrm{~nm}$ at $420 / 410 \mathrm{~nm}$ for excitation using a spectrofluorophotometer (RF-5300PC, Shimadzu Corp., Kyoto, Japan). Fluorescence intensity at $590 \mathrm{~nm}$ for excitation at 420 $\mathrm{nm}$ was regarded as the amount of ZPP formed, and fluorescence intensity at $630 \mathrm{~nm}$ for excitation at $410 \mathrm{~nm}$ was regarded as the amount of PPIX formed. All operations were carried out in darkness as much as possible.

\subsection{Quantitative analysis of ZPP, PPIX and heme}

116 The contents of ZPP, PPIX and heme were determined by HPLC as described by Guo, 117 Lim \& Peters (1991) with some modification. Porphyrins were extracted by acetic 118 acid/acetate ethyl (1:4, v/v) as described by Smith, Doran, Mazur \& Bush (1980) with 119 some modification. One milliliter of model solution was mixed vigorously with $9 \mathrm{ml}$ of 120 acetic acid/acetate ethyl (1:4, v/v), and the mixture was held on ice for $30 \mathrm{~min}$. After 121 centrifugation (3,000 rpm, $15 \mathrm{~min})$, the supernatant was collected. This extraction 122 operation was carried out twice. The resulting solution was mixed with an equal 123 volume of methanol/ammonium acetate (86:14, v/v, $\mathrm{pH} 5.16)$. The sample was filtered 124 through a $0.45-\mu m$ filter (Minisart RC4, Sartorius AG, Goettingen, Germany). An STR 125 ODS-II column (4.6 x 150 mm, Shinwa Chemical Industries Ltd., Kyoto, Japan) was 126 used for separation of porphyrin, and the separation was carried out by isocratic elution 127 using methanol/ammonium acetate ( $86: 14, \mathrm{v} / \mathrm{v}, \mathrm{pH} 5.16)$ at a flow rate of $0.6 \mathrm{ml} / \mathrm{min}$ at $12835^{\circ} \mathrm{C}$. Forty microliters of each sample was injected. The detection of ZPP and PPIX was carried out at excitation and emission wavelengths of 420/400 and 590/630 nm, respectively. Heme was monitored at $400 \mathrm{~nm}$ absorption. All operations of extraction 
131 were carried out in darkness as much as possible.

132

133 2.5. Statistical analysis

134

135 Results are expressed as mean values of three independent trials in each individual.

136 Data were analyzed using one-way analysis of variance (ANOVA) with Scheffés test.

137 Statistics were calculated using Microsoft Excel 2002 and a statistic add-in software

138 (Excel Toukei 2002 for Windows, Social Survey Research Information Co., Ltd., Tokyo,

139 Japan). A probability of $P<0.05$ was considered statistically significant. 
142 First, in order to investigate the effects of exogenous myoglobin in our established 143 experimental model, the effect of the amount of added myoglobin on the amount of ZPP 144 formed was examined (Fig. 1). When exogenous myoglobin was added at $0.1 \%$, the 145 a mount of ZPP formed was maximal. With further addition of myoglobin, the amount 146 of formed ZPP gradually decreased. The amount of ZPP formed was significantly 147 increased with the addition of $0.1 \%$ exogenous myoglobin. The cause of the increase is 148 unclear and it may be because myoglobin used in this study was metmyoglobin or 149 included impurities. However, ZPP was formed despite no addition of myoglobin. 150 Therefore, this result suggested that exogenous myoglobin is not always essential for 151 the formation of zinc protoporphyrin IX and ZPP. It is also possible that ZPP is formed only from pork components. Since the addition of exogenous myoglobin in a model 153 solution might make it difficult to elucidate the mechanism by which ZPP is formed, the 154 mechanism should be investigated in a simpler model system with no addition of 155 exogenous myoglobin. However, the cause of increase in ZPP formation by the addition 156 of $0.1 \%$ exogenous myoglobin was not verified.

157 Next, we established a simpler model system composed of pork homogenate and 158 antibiotics without addition of exogenous myoglobin and investigated the effects of 159 various factors on ZPP formation (Fig. 2). Oxygen inhibited ZPP formation (Fig. 2A) 160 and the amount of ZPP formed was increased with increase in the amount of pork added 161 (Fig. 2B). The amount of ZPP formed rapidly increased during the first 3 days of 162 incubation and increased slightly after the third day (Fig. 2C). The amount of ZPP 163 formed was also increased with increase in incubation temperature within the range of 164 temperatures examined $\left(4,15,25\right.$ and $\left.35^{\circ} \mathrm{C}\right)$ (Fig. 2D). The amount of ZPP formed 165 peaked at about $\mathrm{pH} 5.5$ and decreased considerably at lower or higher pH (Fig. 2E). 
166 The results showing that ZPP formation depended on many factors suggested the 167 involvement of an enzyme. The most notable dependence is the $\mathrm{pH}$ dependence. ZPP 168 formation peaked at 5.5, which is very close to the ultimate $\mathrm{pH}$ of meat. The optimal $169 \mathrm{pH}$ range was narrower than those for general enzymes. Although the cause is not 170 clear, this $\mathrm{pH}$ range might be an important clue to elucidate the mechanism by which $171 \quad \mathrm{ZPP}$ is formed.

172 On the other hand, it has been reported that little ZPP was present in meat products 173 cured with nitrite and/or nitrate (Adamsen Møller, Laursen, Olsen \& Skibsted, 2006). 174 Therefore, the influence of added nitrite was investigated by using the model system 175 (Fig. 2F). The formation of ZPP was inhibited significantly by addition of nitrite of 176 more than $10 \mu \mathrm{M}(0.69 \mathrm{mg} / \mathrm{L})$. Since the concentration at which ZPP formation was 177 inhibited was much lower than the amount of nitrite added to common meat products, it 178 was speculated that low ZPP content in meat products cured with nitrite and/or nitrate 179 is caused by the inhibition of ZPP formation by nitrite. However, the cause is still 180 unclear and future studies are needed.

181 In previous studies (Wakamatsu, Nishimura \& Hattori, 2004; Wakamatsu, Okui, 182 Nishimura \& Hattori, 2004), the amount of ZPP formed was determined by fluorescence 183 intensity and was not determined quantitatively. Heme content was also not 184 measured. Therefore, we determined the quantities of ZPP and heme in the model 185 solutions. As shown in Fig. 3, although the amount of ZPP had significantly increased 186 after 5-day incubation, a decrease in the amount of heme was not observed. PPIX was 187 not detected before and after incubation. Therefore, the total a mount of ZPP and heme 188 was increased compared with the total amount before incubation. If ZPP was just 189 formed from heme with substitution of $\mathrm{Zn}$ for Fe, the sum total amount of heme and 190 ZPP would not be increased. This result therefore suggested that ZPP was not formed 191 by $\mathrm{Fe}-\mathrm{Zn}$ substitution in heme but was independently formed in the model during 192 incubation. At the last step of heme biosynthesis, heme was formed by inserting Fe 
into PPIX (Ferreira, 1999; Dailey, Dailey, Wu, Medlock, Rose \& Wang, 2000; Dailey, 2002). It is therefore possible that $\mathrm{ZPP}$ is formed by insertion of $\mathrm{Zn}$ into PPIX generated independently. Thus, the chelation of zinc by a chelator was predicted to inhibit the formation of ZPP and to result in the accumulation of PPIX.

197 Next, we observed the fluorescence spectrum of acetone extract after incubation in the model solution with addition of EDTA, a bivalent metal chelator (Fig. 4). A strong peak at $630 \mathrm{~nm}$ was observed in the model solution with addition of EDTA. The maximum excitation wavelength of the fluorescence peak was about $410 \mathrm{~nm}$, shorter than the excitation wavelength of ZPP $(420 \mathrm{~nm})$ in agreement with results of a previous study (Shepherd \& Dailey, 2005). This fluorescence peak coincided with that of PPIX (Shepherd \& Dailey, 2005; Veberg et al., 2006). Thus, since the chelation of zinc by EDTA inhibited the formation of ZPP, ZPP seems to be formed by insertion of Zn into PPIX.

Next, in order to determine whether PPIX was formed from the demetalation of heme or independently, porphyrin contents in the model solutions with and without addition of EDTA were determined after incubation (Fig. 5). Although the addition of EDTA into a model solution significantly inhibited ZPP formation and the amount of PPIX was increased significantly, there was no change in heme content $(P>0.05)$. The increase in PPIX content and the fact that the amount of heme did not change strongly suggested that PPIX was formed not from heme but from another substrate. EDTA was added into a model solution, but only a small amount of ZPP was present in the solution. This is due to the presence of ZPP originally contained in pork (see Fig. 3).

215 We also investigated the effects of various factors on the formation of PPIX in simple model systems with addition of EDTA. Oxygen tended to inhibit the formation of PPIX

217 (Fig. 6A). PPIX was increased with increase in pork content in model solutions (Fig. 
increased with increase in incubation temperature (Fig. 6D) and was maximal at about

$221 \mathrm{pH} 5.5$ (Fig. 6E). The addition of more than $10 \mu \mathrm{M}$ of nitrite inhibited ZPP formation significantly (Fig. 6F). All of these patterns of PPIX formation are very similar to those of ZPP formation (Fig. 2). Thus, these results indicated that ZPP in a model solution was formed by the insertion of $\mathrm{Zn}$ into PPIX, which was formed not from heme but independently. It is therefore possible that ZPP is also formed by the same pathway in Parma ham. PPIX is formed from protoporphyrinogen IX (proto'gen) by protoporphyrinogen oxidase (PPO; EC 1.3.3.4) in the he me biosynthesis system (Dailey, 2002). The enzyme activity of mammalian PPO has been shown to be maximal at $\mathrm{pH}$ 8.6 to 8.7 (Poulson, 1976), much higher than results obtained in the present study. On the other hand, the inhibition of PPIX formation by oxygen was reduced in model systems with addition of EDTA, but there was no significant difference between them. There are two forms of PPO, an oxygen-dependent form and an oxygen-independent 233 form (Dailey, 2002), and they may contribute to PPIX formation. Our results revealed 234 that nitrite inhibited the formation of not only ZPP but also PPIX. Anaerobic oxidation of proto'gen to PPIX has been demonstrated in extracts of several microorganisms by nitrite or nitrate as an electron acceptor (Jacobs \& Jacobs, 1976; Klemm \& Barton, 1985). Since the inhibition of PPIX formation by nitrite is not still clear, further studies are needed to elucidate the contribution of proto'gen and PPO to ZPP/PPIX formation. The patterns of ZPP and PPIX formation were similar in model systems. The $\mathrm{pH}$ curves with maximum peaks at about 5.5 are noteworthy, and they might serve as a clue to elucidating the mechanism by which ZPP/PPIX is formed.

Furthermore, our results revealed that nitrite inhibited the formation of not only 243 ZPP but also PPIX. Color formation in cured meat products is caused mainly by the 244 reaction of endogenous myoglobin in meat with nitric oxide (NO) (Sakata, 2000; Fox, 245 1966). NO is produced from added nitrite (or nitrate) as the curing agent. NO has a 246 high affinity for non-heme iron proteins (Fujii \& Yoshimura, 1996) and copper proteins 
247 (Suzuki, 1996). NO is a paramagnetic molecule and has a high affinity for metal ions 248 other than heme (Fujii and Yoshimura, 1996). Consequently, many metalloenzymes 249 are inactivated by NO. Iron-sulfur $(\mathrm{Fe}-\mathrm{S})$ proteins have a very high affinity for NO. 250 Mammalian ferrochelatase contains a [2Fe-2S] cluster (Ferreira, Franco, Lloyd, Per eira, 251 Moura, Moura, \& Huynh, 1994; Dailey, Dailey, Wu, Medlock, Rose \& Wang, 2000), and 252 the cluster is labile and sensitive to destruction by NO (Sellers, Johnson, \& Dailey, 253 1996). It has been reported that ferrochelatase as a zinc chelatase participate in ZPP 254 formation from myoglobin in pork loin extract and porcine heart extract (Ishikawa, 255 Yoshihara, Baba, Kawabuchi, Sato, Numata \& Matsumoto, 2006a; Ishikawa, Yoshihara, 256 Baba, Kawabuchi, Sato, Numata \& Matsumoto, 2006b). Although the participation of 257 ferrochelatase is still not clear, the inactivation of metalloenzyme by NO may be 258 involved in the inhibition of PPIX formation.

259 The results of this study might serve as a clue to elucidating the mechanism by which 260 ZPP/PPIX is formed. Since the formation of PPIX had a strong influence on that of ZPP, 261 it is necessary to investigate the steps prior to PPIX formation in more detail. 
This study demonstrated that ZPP in a model solution was not formed by $\mathrm{Fe}-\mathrm{Zn}$ substitution in heme but was formed by the insertion of $\mathrm{Zn}$ into PPIX, which was

267 formed independently. It was shown that ZPP formation was strongly influenced by 268 PPIX formation. The fact that there is little ZPP in cured meat products is because ZPP cannot be formed as a result of inhibition of PPIX formation by nitrite. A full understanding of ZPP formation in Parma ham awaits elucidation of the formation of

271 PPIX or its precursor.

Acknowledgments

275 This study was supported partially by a Grant-in-Aid for Scientific Research 276 (16780184) from the Ministry of Education, Culture, Sports, Science and Technology of 277 Japan.

278 
Adamsen, C. E., Møller, J. K. S., Laursen, K., Olsen, K. \& Skibsted, L.H. (2006). Zn-porphyrin formation in cured meat products: Effect of added salt and nitrite, Meat Science, 72, 672-679.

Dailey H. A. (2002). Terminal steps of haem biosynthesis, Biochemical Society Transactions, 30, 590-595.

Dailey, H. A., Dailey, T. A., Wu, C.-K.,. Medlock, A. E, Rose, J. P., \& Wang, K.-F. (2000). Ferrochelatase at the millennium: structures, mechanisms and [2Fe-2S] clusters, Cellular \& Molecular Life Sciences, 57, 1909-1926.

Ferreira, G. C., Franco, R., Lloyd, S. G., Pereira, A. S., Moura, I., Moura, J. J., \& Huynh, B. H. (1994) Mammalian ferrochelatase, a new addition to the metalloenzyme family. The Journal of Biological Chemistry, 269, 7062-7065.

Ferreira, G.C. (1999) Ferrochelatase. The International Journal of Biochemistry \& Cell Biology, 31, 995-1000.

Fox J.B. Jr. (1966) The chemistry of meat pigments, Journal of Agricultural and Food Chemistry, 14, 207-210.

Fujii , S. \& Yoshimura, T. (1996) (Japanese) 13 Interaction of nitric oxide with non-heme iron proteins,

Guo, R., Lim, C. K., \& Peters T. J. (1991). High-perfor mance liquid chromatographic assays for protoporphyrinogen oxidase and ferrochelatase in human leucocytes, Journal of Chromatography, 566, 383-396.

Hornsey, H. C. (1956). The colour of cooked cured pork. I. Estimation of the nitric oxide-heam pig ments. Journal of the Science of Food and Agriculture, 7, 534-540.

Ishikawa, H., Yoshihara, M., Baba, A., Kawabuchi, T., Sato, M., Numata, M. \& Matsumoto, K. (2006a). Formation of zinc protoporphyrin IX from myoglobin with pork loin extract, Journal of The Faculty of Agriculture Kyusyu University, 51, 
93-97.

Ishikawa, H., Yoshihara, M., Baba, A., Kawabuchi, T., Sato, M., Numata, M. \& Matsumoto, K. (2006b). Formation of zinc protoporphyrin IX from myoglobin in porcine heart extract, Food Science and Technology Research, 12, 125-130.

Jacobs, N. J., \& Jacobs, J. M. (1976). Nitrate, fumarate, and oxygen as electron acceptors for a late step in microbial heme synthesis, Biochimica et Biophysica Acta, $449,1-9$

Klemm, D. J., \& Barton, L. L. (1985). Oxidation of protopor phyrinogen in the obligate anaerobe Desulfovibrio gigas, Journal of Bacteriology, 164, 316-320.

Møller, J. K. S., Adamsen, C. E., \& Skibsted, L. H. (2003). Spectral characterization of red pigment in Italian-type dry-cured ham. Increasing lipophilicity during processing and maturation. European Food Research and Technology, 216, 290-296.

Møller, J. K. S., Adamsen, C. E., Catharino, R. R., Skibsted, L. H., \& Eberlin M. N. (2007) Mass spectrometric evidence for a zinc-porphyrin complex as the red pigment in dry-cured Iberian and Parma ham. Meat Science, 75, 203-210.

Morita, H., Niu, J., Sakata, R., \& Nagata, Y. (1996). Red pigment of Parma ham and bacterial influence on its formation. Journal of Food Science, 61, 1021-1023.

Okayama, T., \& Nagata, Y. (1978). (Japanese) A Modification of the method for measuring the color formation ability of meat products. Japanese Journal of Zootechnical Science, 49, 866-871.

Okayama, T, \& Nagata, Y. (1979). (Japanese) A method for the determination of total heme pigments in meat products. Japanese Journal of Zootechnical Science, 50, $15-21$.

Parolari, G., Gabba, L., \& Saccani, G. (2003). Extraction properties and absorption spectra of dry cured hams made with and without nitrate. Meat Science, 64, 483-490.

Poulson, R. (1976). The enzymic conversion of protoporphyrinogen IX to protoporphyrin 
IX in mammalian mitochondria, The Journal of Biological Chemistry, 251, 3730-3733.

Sakata, R. (2000) Studies on physicochemical characteristics of red pigments in meat products. Animal Science Journal, 71, 1-16.

Sellers, V. M., Johnson, M. K., \& Dailey, H. A. (1996) Function of the [2Fe-2S] cluster in mammalian ferrochelatase: a possible role as a nitric oxide sensor. Biochemistry, 35, 2699-2704.

Shepherd, M., \& Dailey, H.A. (2005). A continuous fluorimetric assay for protopor phyrinogen oxidase by monitoring porphyrin accumulation, Analytical Biochemistry, 344, 115-121.

Smith, R. M., Doran, D., Mazur M., \& Bush, B (1980). High-Performance Liquid Chromatographic Determination of Protoporphyrin and Zinc Protoporphyrin in Blood, Journal of Chromatography, 181, 319-327.

Toldrá, F. (2002). Dry-cured meat products, Connecticut, USA: Food \& Nutrition Press, Inc.

Veberg, A., Sørheim, O., Moan, J., Iani, V., Juzenas, P., Nilsen, A. N., \& Wold, J. P. (2006). Measurement of lipid oxidation and porphyrins in high oxygen modified atmosphere and vacuum-packed minced turkey and pork meat by fluorescence spectra and images. Meat Science, 73, 511-520.

Wakamatsu, J., Nishimura, T., \& Hattori, A. (2004). A Zn-porphyrin complex contributes to bright red color in Parma ham. Meat Science, 67, 95-100.

Wakamatsu, J., Okui, J., Ikeda, Y., Nishimura, T., \& Hattori, A. (2004). Establishment of a model experiment sy stem to elucidate the mechanism by which Zn-protoporphyrin IX is formed in nitrite-free dry-cured ham. Meat Science, 68, 313-317.

Wakamatsu, J., Odagiri, H., Nishimura, T., \& Hattori, A. (2006). Observation of the distribution of Zn protoporphyrin IX in Parma ham by using purple LED and image 

analysis. Meat Science., 74, 594-599.

361 Wakamatsu, J., Ito, T., Nishimura, T., \& Hattori A. (2007). Direct demonstration of the 362 presence of zinc in the acetone-extractable red pigment from Parma ham. Meat $363 \quad$ Science., $76,385-387$. 
Figure legends

Fig. 4. Fluorescence pattern of acetone extract of model solutions with (broken line, Ex.

Fig. 3. Heme ( $(\mathbf{)})$ and $\mathrm{ZPP}(\varpi)$ concentrations in model solutions without addition of exogenous myoglobin before and after incubation. Porphyrins extracted by acetic acid/acetic ethyl were measured by HPLC. Bars represent the standard deviation of the means (n=3). n.s.: not significant (vs before incubation). $410 \mathrm{~nm}$ ) and without (solid line, Ex. $420 \mathrm{~nm}$ ) addition of EDTA after 5 days at $25^{\circ} \mathrm{C}$.

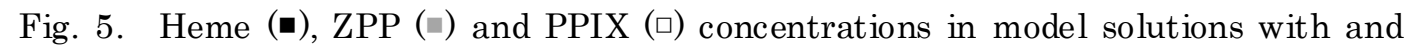


389 without addition of EDTA after 5 days at $25^{\circ} \mathrm{C}$. Porphyrins extracted by acetic 390 acid/acetic ethyl were measured by HPLC. Bars represent the standard deviation 391 of the means ( $n=3)$. n.s.: not significant (vs with addition of EDTA).

393 Fig. 6. Effects of oxygen (A), pork content (B), incubation day (C), temperature (D), pH 394 (E) and nitrite (F) on the formation of ZPP in the model solution with addition of 395 EDTA. The fluorescence intensity of acetone extracts was measured after 396 incubation. Bars represent the standard deviation of the means $(n=3)$. abcde: 397 Values in the same examination bearing different letters are significantly different $398 \quad(P<0.05) . \quad$ n.s.: not significant.

399

400 Table 1. Experimental designs by using a model system 401 


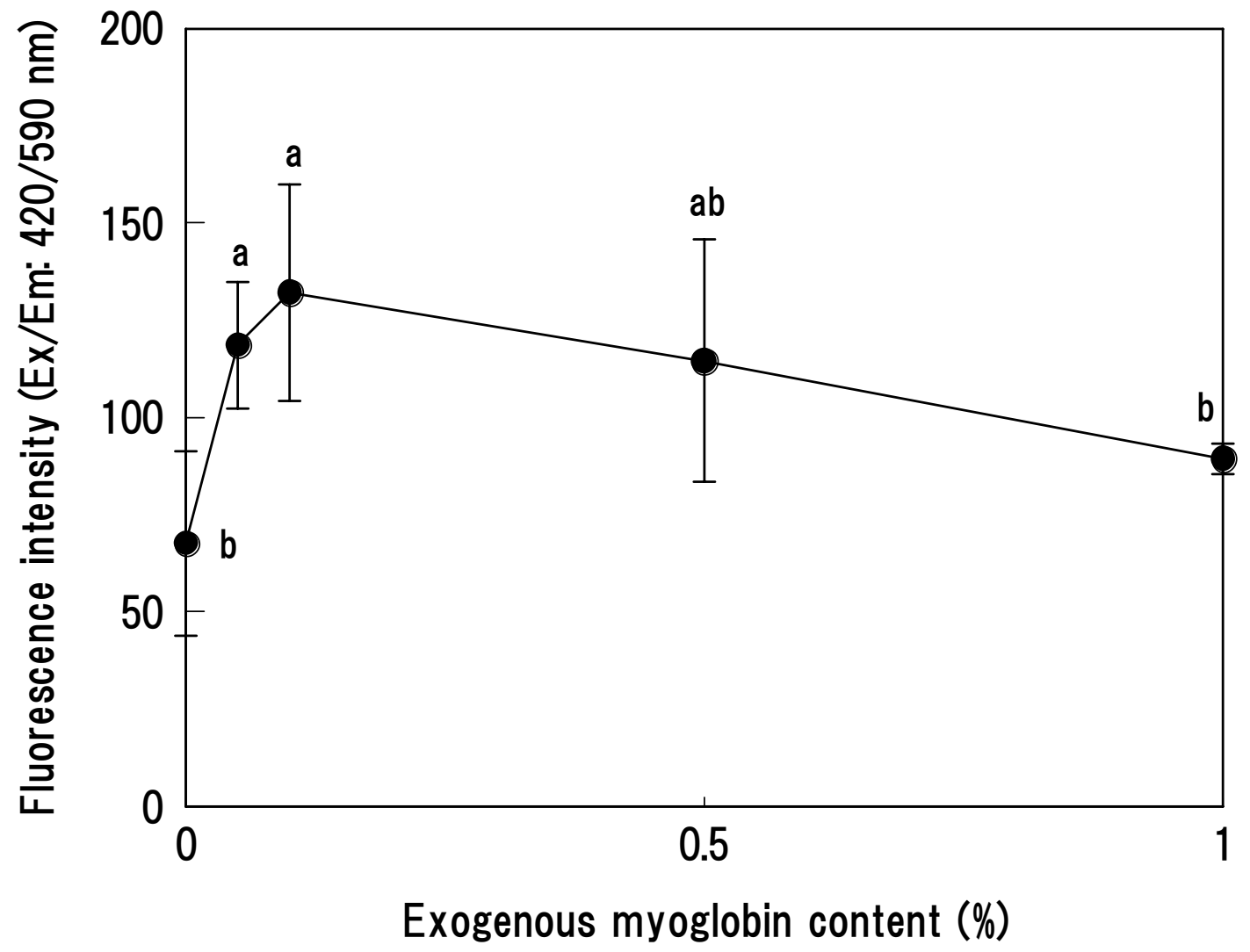

Fig.1 

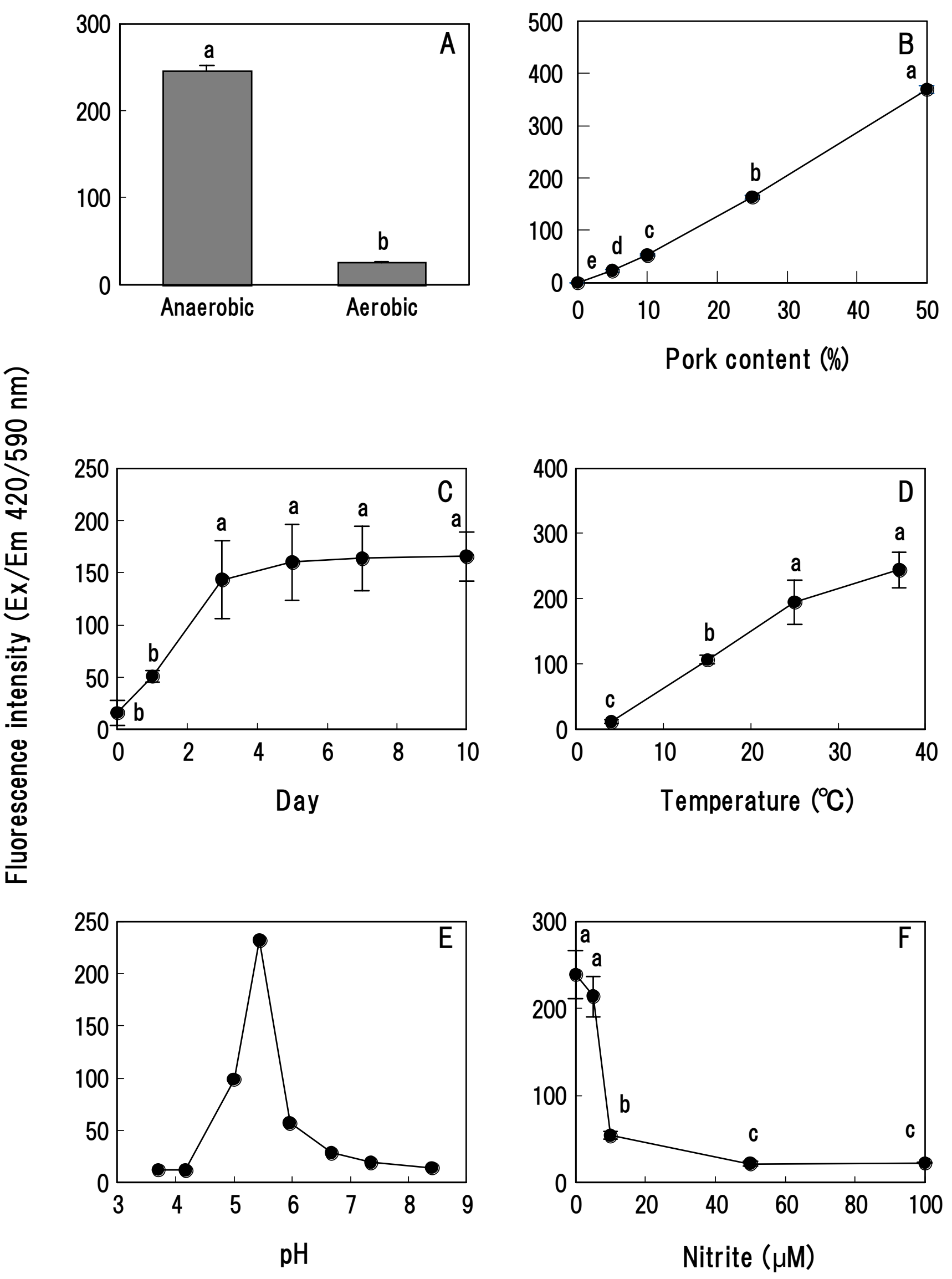

Fig.2 
Figure3

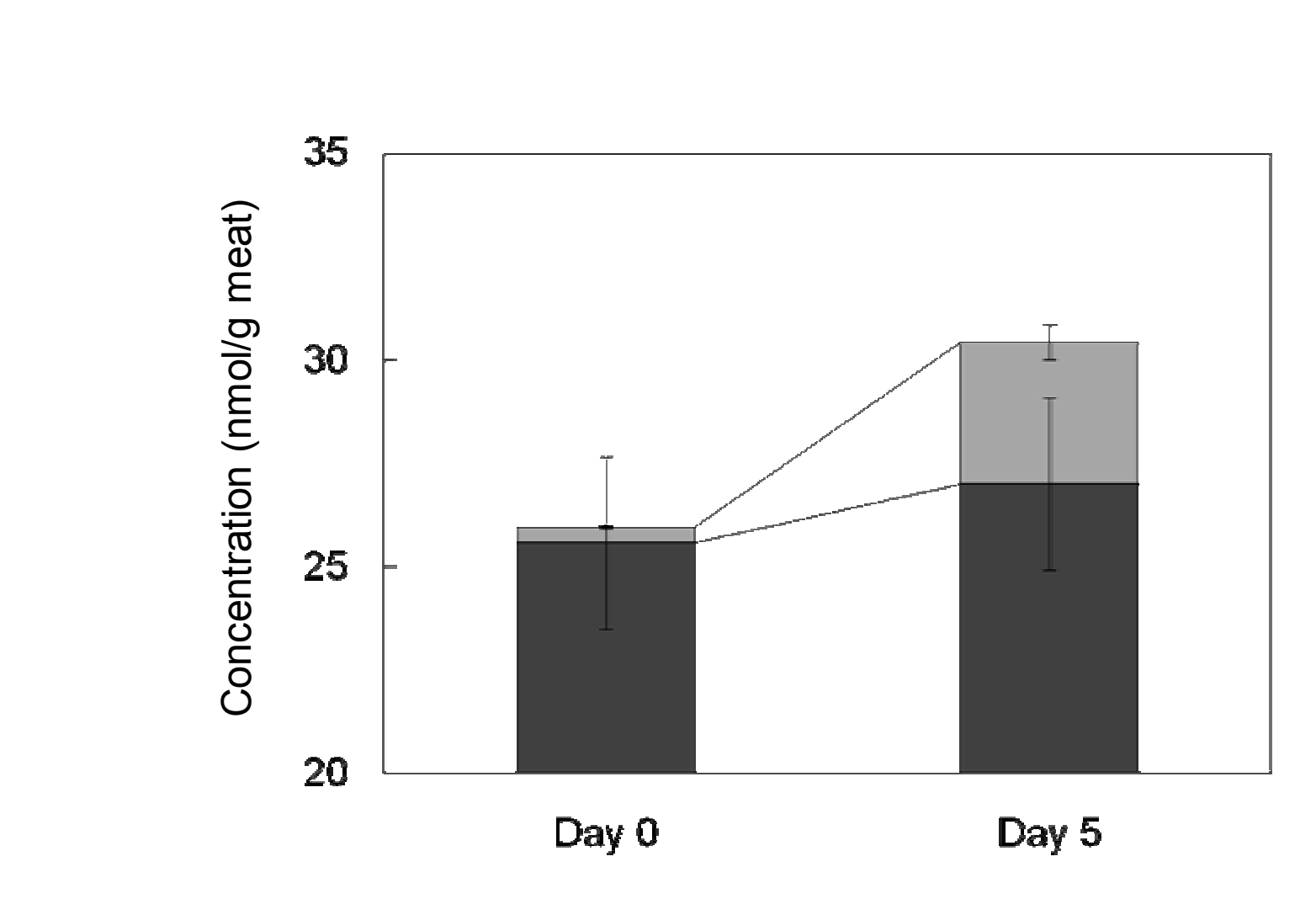

Fig. 3 3

gure3

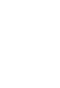




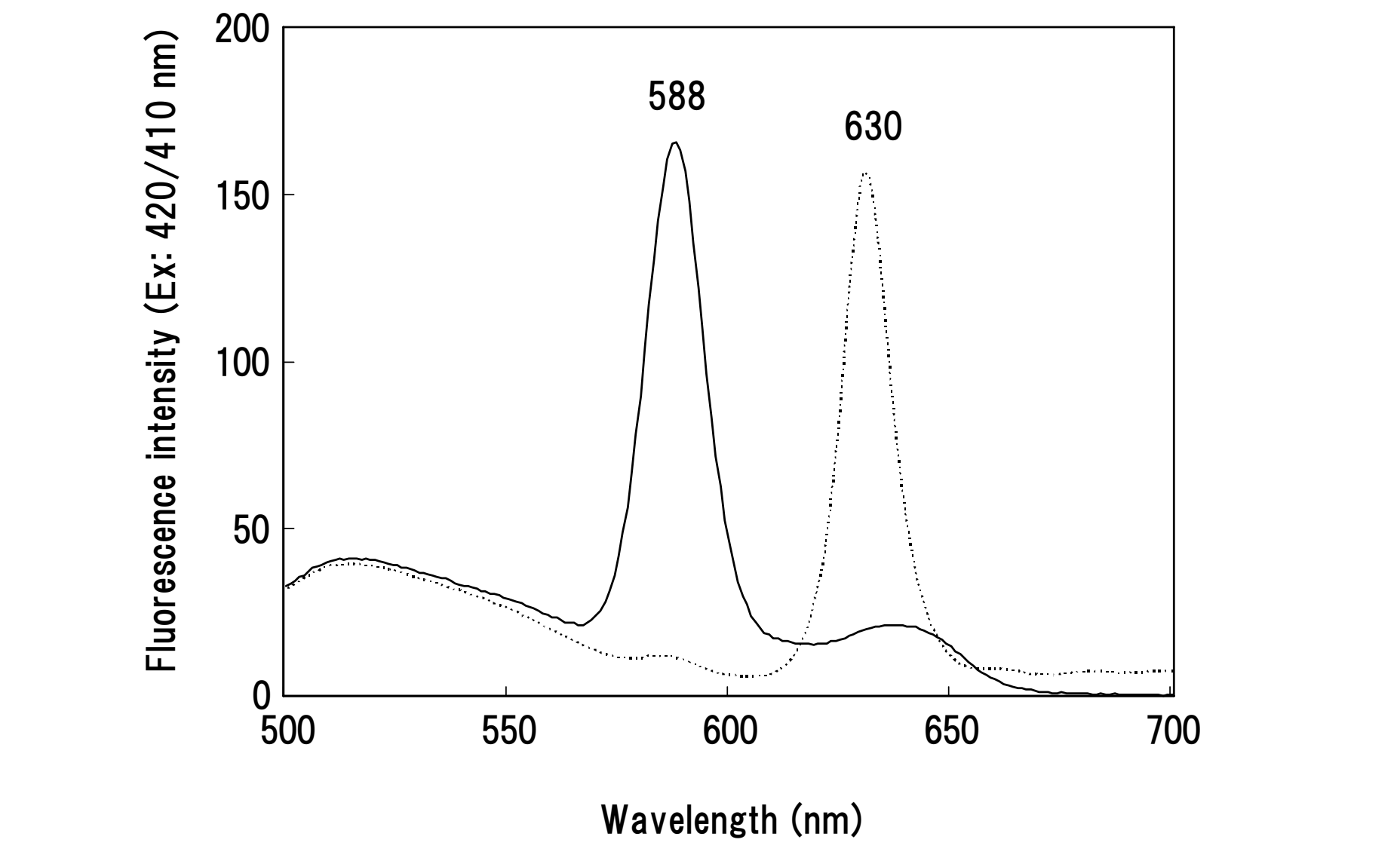

Fig.4

\section{Fir}




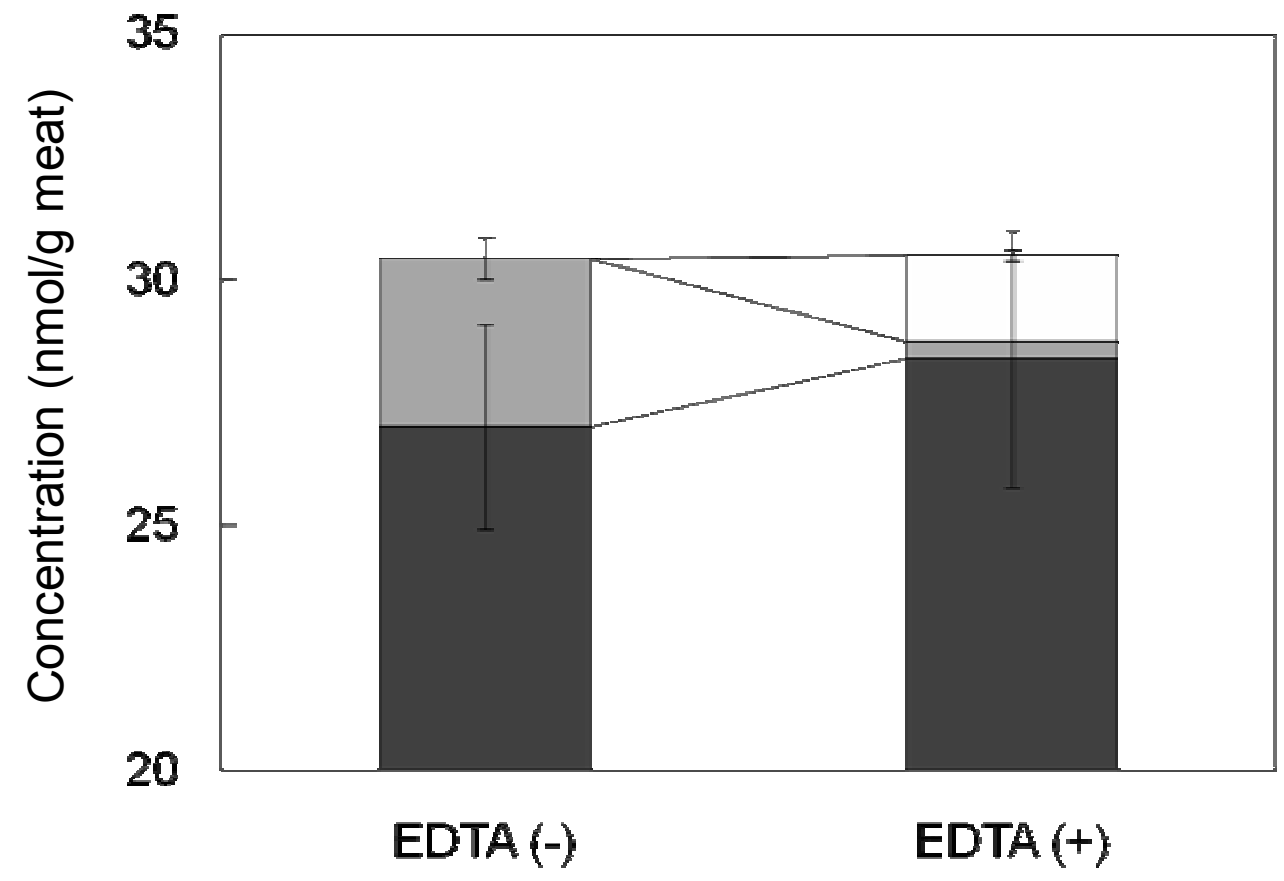

Fig. 5 

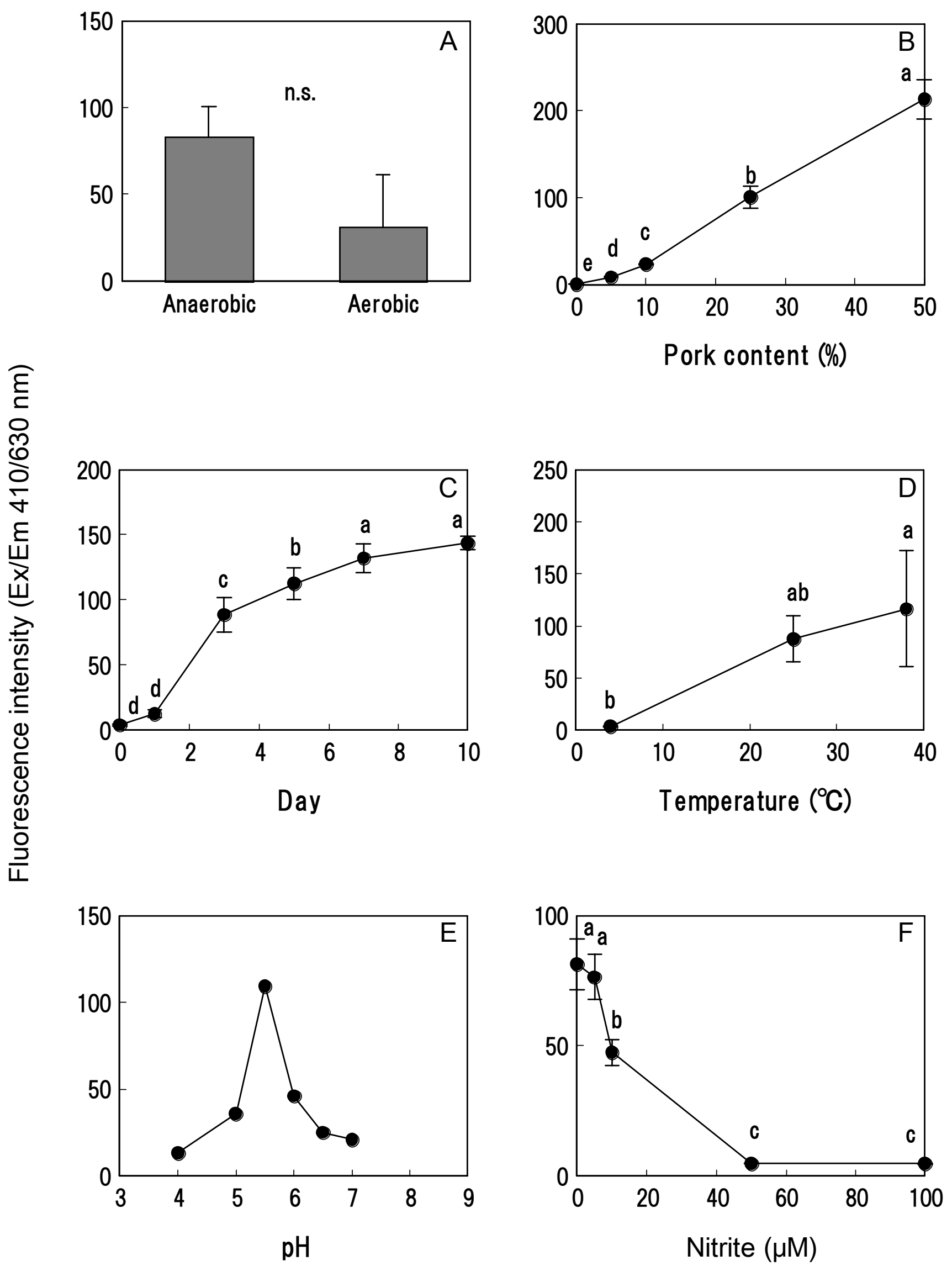

Fig.6 
Table 1. Experimental designs by using a model system

\begin{tabular}{|c|c|c|c|c|c|c|c|}
\hline Pork & Myoglobin & EDTA & Oxygen & Temperature & Day & $\mathrm{pH}$ & Nitrite \\
\hline \multicolumn{8}{|c|}{ Experimental model designs with addition of exogenous myoglobin } \\
\hline $20 \%$ & $0-1 \%{ }^{a}$ & - & $<0.1 \%$ & $25^{\circ} \mathrm{C}$ & 5 & - & - \\
\hline \multicolumn{8}{|c|}{ Experimental model designs without addition of exogenous myoglobin } \\
\hline $20 \%$ & - & - & $<0.1 \%$ & $25^{\circ} \mathrm{C}$ & 5 & - & - \\
\hline $20 \%$ & - & - & $N A^{b}$ & $25^{\circ} \mathrm{C}$ & 5 & - & - \\
\hline $0-50 \%{ }^{c}$ & - & - & $<0.1 \%$ & $25^{\circ} \mathrm{C}$ & 5 & - & - \\
\hline $20 \%$ & - & - & $<0.1 \%$ & $25^{\circ} \mathrm{C}$ & $0-10^{d}$ & - & - \\
\hline $20 \%$ & - & - & $<0.1 \%$ & $4-37^{\circ} \mathrm{C}^{\mathrm{e}}$ & 5 & - & - \\
\hline $20 \%$ & - & - & $<0.1 \%$ & $25^{\circ} \mathrm{C}$ & 5 & $3.5-8.5$ & - \\
\hline $20 \%$ & - & - & $<0.1 \%$ & $25^{\circ} \mathrm{C}$ & 5 & - & $0-100 \mu M^{f}$ \\
\hline \multicolumn{8}{|c|}{ Experimental model designs in the chelation on bivalent metals by EDTA } \\
\hline $20 \%$ & - & $0.5 \mathrm{mM}$ & $<0.1 \%$ & $25^{\circ} \mathrm{C}$ & 5 & - & - \\
\hline $20 \%$ & - & $0.5 \mathrm{mM}$ & $N A^{b}$ & $25^{\circ} \mathrm{C}$ & 5 & - & - \\
\hline $0-50 \%{ }^{c}$ & - & $0.5 \mathrm{mM}$ & $<0.1 \%$ & $25^{\circ} \mathrm{C}$ & 5 & - & - \\
\hline $20 \%$ & - & $0.5 \mathrm{mM}$ & $<0.1 \%$ & $25^{\circ} \mathrm{C}$ & $0-10^{d}$ & - & - \\
\hline $20 \%$ & - & $0.5 \mathrm{mM}$ & $<0.1 \%$ & $4,25,37^{\circ} \mathrm{C}$ & 5 & - & - \\
\hline $20 \%$ & - & $0.5 \mathrm{mM}$ & $<0.1 \%$ & $25^{\circ} \mathrm{C}$ & 5 & $3.5-8.5$ & - \\
\hline $20 \%$ & - & $0.5 \mathrm{mM}$ & $<0.1 \%$ & $25^{\circ} \mathrm{C}$ & 5 & - & $0-100 \mu M^{f}$ \\
\hline
\end{tabular}

Antibiotics were added to all model solutions to final concentrations of $100 \mathrm{units} / \mathrm{ml}$ for penicillin $\mathrm{G}$ potassium, $0.1 \mathrm{mg} / \mathrm{ml}$ for streptomycin sulfate and $0.05 \mathrm{mg} / \mathrm{ml}$ for gentamicin sulfate.

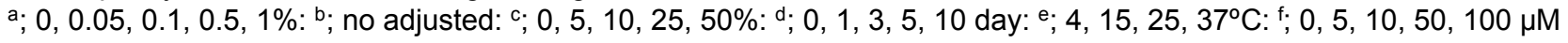

